Provided by the author(s) and University of Galway in accordance with publisher policies. Please cite the published version when available.

\begin{tabular}{|c|l|}
\hline Title & Four Heuristics to Guide Structured Content Crawling \\
\hline Author(s) & $\begin{array}{l}\text { Umbrich, Jürgen; Harth, Andreas; Hogan, Aidan; Decker, } \\
\text { Stefan }\end{array}$ \\
\hline $\begin{array}{c}\text { Publication } \\
\text { Date }\end{array}$ & 2008 \\
\hline $\begin{array}{c}\text { Publication } \\
\text { Information }\end{array}$ & $\begin{array}{l}\text { Jürgen Umbrich, Andreas Harth, Aidan Hounan, Stefan Decker } \\
\text { International Conference on Web Engineering (Short Paper), } \\
\text { 2008. }\end{array}$ \\
\hline Item record & http://hdl.handle.net/10379/421 \\
\hline
\end{tabular}

Downloaded 2023-04-26T08:51:54Z

Some rights reserved. For more information, please see the item record link above.

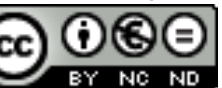




\title{
Four Heuristics to Guide Structured Content Crawling
}

\author{
Jürgen Umbrich and Andreas Harth and Aidan Hogan and Stefan Decker \\ National University of Ireland, Galway \\ Digital Enterprise Research Institute \\ \{firstname.lastname@deri.org\}
}

\begin{abstract}
Search engines focusing on particular media types face difficulties in discovering suitable URIs on the Web. Since the engines are only interested in a small fraction of the Web, a crawler should use heuristics to concentrate on that fraction. To devise such a heuristic, we postulate four hypotheses based on RFCs and W3C recommendations to find cues for certain content types. Tests on a corpus of $22 \mathrm{~m}$ files (793GB content size) containing 630m URIs show that for the content types text, image, and application, the recommendations are mostly being followed, while results for audio and video are much less consistent. Our findings and recommendations can be implemented as heuristics for efficient discovery of structured content on the Web on top of existing crawlers.
\end{abstract}

\section{Introduction}

While established search engines focus on hypertext documents, in recent years a number of specialised search engines have emerged that collect and integrate information from files of particular media types. Seeqpod ${ }^{1}$ and Blinkx ${ }^{2}$ offer search over audio and video files, Google Scholar ${ }^{3}$ and CiteSeer ${ }^{4}$ are digital libraries of printable documents, Technorati ${ }^{5}$ provides real-time access to news-feeds, and Seekda ${ }^{6}$ offers search capabilities for web services. A new generation of search engines with powerful query functionality are emerging such as $\mathrm{SWSE}^{7}, \mathrm{Swoogle}^{8}$, Watson ${ }^{9}, \mathrm{Fal}-$

\footnotetext{
${ }^{1}$ http://www.seeqpod.com/

${ }^{2}$ http://www.blinkx.com/

${ }^{3}$ http://scholar.google.com/

${ }^{4} \mathrm{http}: / /$ citeseer.ist.psu.edu/

${ }^{5} \mathrm{http} / / /$ technorati.com/

${ }^{6} \mathrm{http}: / /$ seekda.com/

${ }^{7}$ http://swse.org/

${ }^{8} \mathrm{http}: / /$ swoogle.umbc.edu/

${ }^{9} \mathrm{http} / / /$ watson.kmi.open.ac.uk/WatsonWUI/
}

consearch $^{10}$ and Sindice ${ }^{11}$, which concentrate on semantic web data. Common to all these specialised search engines is that they rely only on documents of specialised media types.

A common issue for targeted search engines is how to discover files of a certain media type on the Web [12]. Most of the search engines provide users with a form or an API to encourage submissions of URIs. In addition they use general Web search engines, like Google, MSN or Yahoo, to harvest URIs using APIs and query constructs like filetype or originurlextension, but public APIs are restricted by result size and invocation frequency Furthermore, the query constructs only enable searching for a specified file extension instead of querying for the media type of the URIs. Since these methods do not provide enough URIs, all the search engines must invest extra effort traversing the Web in order to find additional sources.

A naïve approach is to use a breadth-first crawler and fetch the connected Web, starting from a seed set of URIs. Search engines in 2005 indexed approximately 11.5 billion documents [8]. In February 2008, the indexed Web was estimated to consist of 45 billion documents ${ }^{12}$. Given that web-scale crawlers can fetch in the order of thousands of pages per second [2], downloading the entire connected Web would take years and require large amounts of CPU power, network bandwidth and storage space. Also, published studies showed that over $90 \%$ of the documents on the Web are hypertext files [14] [7]. Therefore, a search engine for a particular media type is focused only on a small subset of the entire Web. Ideally a specialised crawler would download only the relevant portion of the Web and would save time and resources compared to the naive approach.

Crawling for HTML documents of a particular topic of interest is related to the problem addressed by focused crawling strategies. Focused crawling, as described in [11], [4] or [3], uses decision rules based on content analysis, link structure and anchor text to keep the crawler focused on a

\footnotetext{
${ }^{10} \mathrm{http}: / / \mathrm{www}$. falconsearch.com/

${ }^{11} \mathrm{http}: / /$ sindice.com/

${ }^{12} \mathrm{http}: / /$ www.worldwidewebsize.com/
} 
specific topic, such as "cycling" or " $H I V$ ", to make better use of crawling resources. In contrast, a web crawler for media type targeted search engines is focused on the document formats (such as audio and video) instead of the topic covered by the documents (e.g. "HIV"). The challenge we address is how to develop a theory of focused crawling for particular media types.

Our main idea is to use information encoded in URI's and in the document structure to uncover cues leading to files with certain media types that we can incorporate into the crawling process. Specifically, our contributions are as follows:

- We conjecture four hypotheses that are instrumental in building a crawler focused on gathering files of a particular media type, based on the file extension (H1) and path tokens (H2) of a URI, the response header field Content-type (H3) and the position of extracted URIs from HTML documents (H4) (Section 2).

- We test the hypotheses, based on a data representative set of 22 million pages with a total file size of 793.36 GB and present as well comprehensive statistics about data size, response code distribution, and the different media types. (Section 3).

- We discuss the result of the hypotheses and give recommendations, based on precision and recall measures, that can improve the discovery of certain media types on the Web.(Section 4).

To summarise our findings: Crawlers targeting on downloading files of a special media type should inspect file extensions and header fields for the content types text, application and image; for the content type s audio and video crawlers should look at the document tree position. The paper ends with an overview of related work in Section 5 and finally concludes in Section 6.

\section{How to Support the Crawling Process}

In the following section we present our four hypotheses, based on thoughts and rationale regarding crawling focused on downloading files of a specific media type. The core idea is to take cues from the information available to a crawler during the crawling process such as URI, header, and positional information in the document tree. The goal is to avoid fetching documents of undesirable media types. We explain our thoughts based on an example URI ${ }^{13}$ referring to a video from the W3C Video on the Web Workshop in 2007. Our assumption is that by exploiting the identifiers a crawler can target discovery of files of a certain media type.

\footnotetext{
${ }^{13}$ http://www.w3.org/2007/08/video/slides/KidsHealth/Angio.avi
}

Firstly, we introduce the definition of media types. Media types are registered with IANA $^{14}$ and they consist of two parts, the content type and the subtype. Furthermore, most of the registered media types have recommended file extension(s). For our running example the media type is video/x-msvideo, with content type video and subtype $\mathrm{x}-\mathrm{msvideo}$. Please note that media types that have a subtype starting with $\mathrm{x}$ - are not registered with the IANA.

\subsection{Crawling Process}

A typical web crawler consists of a URI queue, a downloading component, a storage or indexing component and a link extraction component. Figure 1 shows the four basic tasks in the crawling process loop. The basic crawling tasks are: 1) Poll a URI from the queue and 2) establish a connection to the server. Therefore, the client sends a HTTP request and receives a HTTP response. Both messages can contain information about the requested or sent media type. The next crawling task is 3) to download the content of the URI and in the last step 4) to extract new URIs from the downloaded files and add them to the URI queue.

Our focus is to discover content type identifying cues before the file content is downloaded, therefore we omit the crawling task 3 ) in the the following sections.

\subsection{Poll URI}

In the first crawling task the available information for a crawler are encoded in the URI. Every network-retrievable document has a Uniform Resource Identifier, called URI, as defined in RFC2396 ${ }^{15}$. A URI consists of five components.

\section{[PROTOCOL]://[HOST]:[PORT]/[PATH][FILE]}

The interesting parts, which can contain information about the media type behind the URI, are the PATH and FILE components: they identify the location of the file on the server. The PROTOCOL component specifies only the access protocol for the file and the HOST and PORT components are the address of the server hosting the file; these components are not dependant on the media type of the content. The file extension is a widely used identifier for the media type behind a URI: web servers often support automatical mappings for static files to Content-type response headers using file extensions. Also, applications typically use file extensions to associate files to application programs. Our URI example has the the FILE component Angio.avi with file extension avi, which is mapped to the media type video/x-msvideo. The mapped media type matches with the real media type of our example. Consequently, this leads us to our first hypothesis:

\footnotetext{
${ }^{14} \mathrm{http} / / / \mathrm{www}$. iana.org/assignments/mime-types/

${ }^{15} \mathrm{http}: / /$ www.ietf.org/rfc/rfc2396.txt
} 


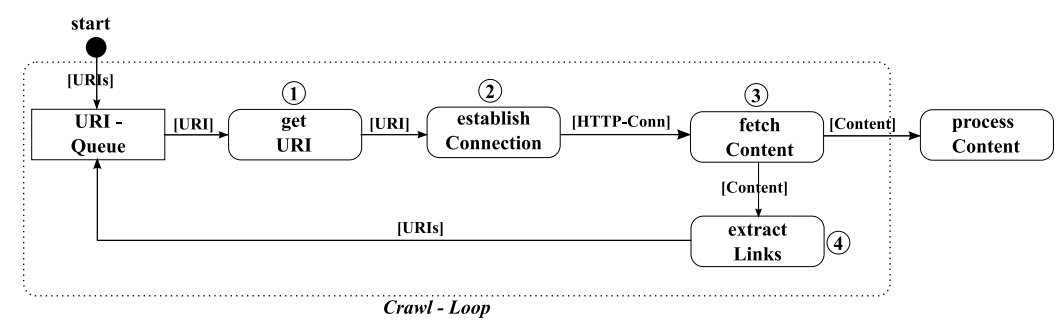

Figure 1. Basic tasks in the crawling process pipeline. Adapted from [15]

\section{H1: The file extension of the FILE part of a URI identi- fies the media type of the file.}

The other interesting component of a URI is the PATH component, which can be part of a hierarchical folder structure. Users organise their content in a folder structure, like storing images in a image directory or videos in a video subfolder, like in our running example. In this case, special subfolders can be used as an indicator for the media types of the documents in this folder. Thus, this leads us to our second hypothesis:

\section{H2: The PATH component of an URI identifies the me- dia type of the file.}

\subsection{Connecting to the Server}

The next task in the crawling process is to establish a connection to the server by sending a request and reading the response. Referring to the HTTP/1.1 specification ${ }^{16}$ web servers should transmit the Content-type and the Content-encoding fields in the response. Sometimes, the mapping from file extension to media type is not configured on the server side: In this case, the server uses the default media type application/octet-stream or omits the Content-type header. For our example URI, the response contains a Content-type header field with the value video/x-msvideo, which matches the real media type of the sample URI. Hence, these thoughts leads us to our third hypothesis:

\section{H3: The Content - type header field identifies the me- dia type of the file.}

\subsection{Extract new URIs}

It is necessary to extract new URIs from the downloaded documents to feed the crawling process loop. For this study, we extract only links from HTML documents. Omitting the textual content of the documents, the available information for a crawler are the position of HTML elements containing

\footnotetext{
${ }^{16}$ http://www.w3.org/Protocols/rfc2616/rfc2616.html
}

links to other files. The HTML 4.0 specification ${ }^{17}$ identifies numerous HTML elements which authors can use to refer to other documents. Beside using navigational links, HTML documents can contain links to meta information, like references to Cascading Style Sheets (CSS) ${ }^{18}$ or RSS feeds in the $<$ LINK $>$ element or links to embedded audio or video files, like in $\angle O B J E C T>$ and $<$ PARAM $>$ elements. Different HTML elements are used to link to files of certain media types; accordingly, this leads us to our fourth hypothesis:

\section{H4: The position of a link in an HTML document iden- tifies the media type of the link target.}

Please note, hypotheses $\mathbf{H 1}$ and $\mathbf{H 3}$ rely on RFC specifications; however, to the best of our knowledge, no analysis exists of how many systems adhere to the specifications. In Section 3.2 we present the results of testing the hypotheses.

\section{Setup and Evaluation}

In this section we describe, firstly, our corpus consisting of $22 \mathrm{~m}$ URIs, the challenge of extracting links and detecting the real media type of a file once downloaded and, secondly, we describe the evaluation of our four hypotheses and present the results.

\subsection{Experimental Setup}

We use URIs from the Open Directory Project (ODP) ${ }^{19}$ and the English Wikipedia ${ }^{20}$ as seed sets. Both sites are human edited and contain collections to external resources and are suitable entry points to relevant files on the Web. We extracted $3.8 \mathrm{~m}$ external links from $\mathrm{ODP}^{21}$ and $6.4 \mathrm{~m}$ external links from Wikipedia ${ }^{22}$ in December 2007. The entire corpus contains $22.2 \mathrm{M}$ documents, $10.2 \mathrm{M}$ derived from the crawler in round one and $12 \mathrm{M}$ in round two; these consist

\footnotetext{
${ }^{17}$ http://www.w3.org/TR/WD-html40-970708/htmlweb.html

${ }^{18}$ http://www.w3.org/Style/CSS/

${ }^{19}$ http://www.dmoz.org/

${ }^{20} \mathrm{http}: / /$ en.wikipedia.org/

${ }^{21} \mathrm{http} / / /$ rdf.dmoz.org/rdf/content.rdf.u8.gz

${ }^{22} \mathrm{http}$ ://download.wikimedia.org/enwiki/
} 


\begin{tabular}{|c|c|c|}
\hline Metric & \multicolumn{2}{|c|}{ Values } \\
\hline Content Size & $793.35 \mathrm{~GB}$ & \\
\hline Mean & $44.13 \mathrm{~KB}$ & \\
\hline Variance & $2.63 \mathrm{~KB}$ & \\
\hline URIs & $22,184,518$ & $(100.00 \%)$ \\
\hline 200 Response Code & $19,030,988$ & $(85.78 \%)$ \\
\hline 404 Response Code & $1,280,608$ & $(5.77 \%)$ \\
\hline Other Response Codes & $1,872,922$ & $(8.44 \%)$ \\
\hline Verified Media Types & $17,230,945$ & \\
\hline Extracted Links & $637,664,510$ & \\
\hline Average Unique Links/Page & 33.5 & \\
\hline
\end{tabular}

Table 1. Metrics about the Corpus

only of links from the ODP seed set. Table 1 shows general metrics about the corpus. The top five top level domains are: .com $(47.92 \%)$,. org $(10.78 \%)$, net $(5.30 \%)$, . uk $(4.92 \%)$ and . de $(4.71 \%)$.

Comparing our corpus to previously published bigger data sets $(815 \mathrm{~m}$ documents, in 2002 [14], and 75m URIs, in 2005 [10]) we can find similar metrics and statistics for the response code, media type (Table 2) and the top level domain distribution. Thus, we assume that our corpus is a valid and comparable data set for our hypotheses test. To download the contents of the seed set URIs we use the crawler framework MultiCrawler [9] and extracted all links from hypertext documents to test hypothesis four $(\mathbf{H 4})$.

We use the latest version of the UNIX tool file (Version 4.23) to verify the media type of the downloaded files. This tool is a standard Unix program for determining the media type of data contained in a file. Deriving the type of a file includes three different tests that are performed in the following order: checking filesystem metadata, checking the magic number of a file, and checking various text characteristics. A disadvantage of the Unix tool file is that it sometimes pools different media types to one single media type, e.g. all different MS Office formats (Word, Excel, PowerPoint) are listed under the media type application/msword. Table 2 lists the top ten media types as determined by file.

\subsection{Evaluation}

In this section we present the results of the test of our four hypotheses. To show a higher level of abstraction, we aggregate the different media types into the content type level and omit the subtypes; this mitigates the shortcomings of the tool file. Table 3 lists the eight different content types and their occurrences in our corpus. The detailed results are available online ${ }^{23}$. Please note, detailed

\footnotetext{
${ }^{23} \mathrm{http} / / /$ sw.deri.org/2008/01/webcontentsurvey/
}

\begin{tabular}{lrr} 
Media Type & Count & Percentage \\
\hline text/html & $14,788,347$ & $85.82 \%$ \\
application/x-gzip & $1,088,940$ & $6.32 \%$ \\
text/xml & 470,487 & $2.73 \%$ \\
text/plain & 323,217 & $1.88 \%$ \\
application/pdf & 200,058 & $1.16 \%$ \\
image/jpeg & 135,520 & $0.79 \%$ \\
text/x-c++ & 72,044 & $0.42 \%$ \\
image/gif & 23,597 & $0.14 \%$ \\
application/x-empty & 23,381 & $0.14 \%$ \\
text/x-c & 16,842 & $0.10 \%$ \\
\hline Total & $17,142,433$ & $99.49 \%$
\end{tabular}

\section{Table 2. Top ten media types detected by the Unix tool file}

\begin{tabular}{llr}
$i$ & Content Type $\left(c_{i}\right)$ & Count $\left(\left|F\left(c_{i}\right)\right|\right)$ \\
\hline 1 & text & $15,677,707$ \\
2 & application & $1,365,397$ \\
3 & image & 166,496 \\
4 & audio & 17,501 \\
5 & video & 2,749 \\
6 & message & 1,044 \\
7 & model & 51 \\
8 & multipart & 0
\end{tabular}

\section{Table 3. Number of content types detected by the Unix tool file}

results for hypothesis two (path tokens) and hypothesis four (link position) are omitted due to space limitations. We use precision and recall measures to quantify the correctness for each of our hypothesis. Table 4 lists the symbols used in verifying the hypotheses.

\subsubsection{H1: File Extension}

Several steps are involved in verifying the extent to which the file extension of a URI identifies the media type of a file. Firstly, we create a mapping between the file extensions and the media types. We start out with a list of common media types ${ }^{24}$ and the corresponding file extensions and synchronise the list with the registered media types at IANA. Secondly, we derive the media type based on file extension, which results in $E\left(c_{i}\right)$. Thirdly, we take the intersection between $F\left(c_{i}\right)$ and $E\left(c_{i}\right)$ which denotes the true positives $T P_{c_{i}}$. Table 5 contains the precision and recall values pertaining to $\mathrm{H} 1$. For computing precision and recall we use: $T P_{c_{i}}=F\left(c_{i}\right) \cap E\left(c_{i}\right), F N_{c_{i}}=F\left(c_{i}\right) \backslash T P_{c_{i}}$

\footnotetext{
${ }^{24} \mathrm{http}: / / \mathrm{www} . w e b m a s t e r-t o o l k i t . c o m / m i m e-t y p e s . s h t m l$
} 


\begin{tabular}{ll} 
Sets & Description \\
\hline$C$ & Content types (see Table 3) \\
$T$ & HTML element positions \\
\hline$F\left(c_{i}\right)$ & URIs of content type $c_{i}$, determined by file \\
$E\left(c_{i}\right)$ & URIs of content type $c_{i}$, determined by file ext. \\
$P\left(p_{i}\right)$ & URIs containing path token $p_{i}$ \\
$H\left(c_{i}\right)$ & URIs with Content - type header field $c_{i}$ \\
$L_{t_{i}}\left(c_{i}\right)$ & URIs from HTML link $t_{i}$ of content type $c_{i}$ \\
\hline$T P_{c_{i}}$ & URIs of content type $c_{i}$ classified as $c_{i}$, \\
$F P_{c_{i}}$ & URIs of content type $c_{j}$ classified as $c_{i}, j \neq i$ \\
$F N_{c_{i}}$ & URIs of content type $c_{i}$ classified as $c_{j}, j \neq i$.
\end{tabular}

Table 4. Symbols and their description used to test the hypotheses

\begin{tabular}{lrrrr}
\multicolumn{1}{c}{$c_{i}$} & \multicolumn{1}{c}{$E\left(c_{i}\right)$} & \multicolumn{1}{c}{$T P_{c_{i}}$} & $P R_{c_{i}}$ & \multicolumn{1}{c}{$R C_{c_{i}}$} \\
\hline text & 5.869 .786 & 4.593 .962 & $78.26 \%$ & $29.30 \%$ \\
appl. & 290.784 & 226.695 & $77.96 \%$ & $16.60 \%$ \\
image & 192.326 & 162.553 & $84.52 \%$ & $97.63 \%$ \\
audio & 28.291 & 15.967 & $56.44 \%$ & $91.23 \%$ \\
video & 9.858 & 2.039 & $20.68 \%$ & $74.17 \%$ \\
message & 179 & 46 & $25.70 \%$ & $4.41 \%$ \\
model & 63 & 51 & $80.95 \%$ & $100.00 \%$ \\
multi. & 1288 & 0 & $0.00 \%$ & -
\end{tabular}

Table 5. Results for H1: File Extension

and $F P_{c_{i}}=E\left(c_{i}\right) \backslash T P_{c_{i}}$. We can see in Table 5 that we derive precision values greater than $50 \%$ for the content types text, application, image, model and audio, which indicates that the file extension, if available, gives a reasonably good estimate of the real content type. For the content types video and message, on the other hand, the file extension is a less reliable indicator for the real content type.

\subsubsection{H2: Path Tokens}

To check if the PATH component of a URI identifies the media type we first split the $P A T H$ component into single path tokens which results in $P\left(p_{i}\right)$. For computing precision and recall we use: $T P_{c_{i}}\left(p_{i}\right)=F\left(c_{i}\right) \cap P\left(p_{i}\right), F N_{c_{i}}\left(p_{i}\right)=$ $F\left(c_{i}\right) \backslash T P_{c_{i}}\left(p_{i}\right)$ and $F P_{c_{i}}\left(p_{i}\right)=P\left(p_{i}\right) \backslash T P_{c_{i}}\left(p_{i}\right)$. Using path tokens to identify content type results generally in high precision values: 29 out of 35 path tokens have a precision value of more than $99 \%$. However, recall is low: the recall for all the path tokens is less than $40 \%$, for the majority of the tokens the recall value is less then $10 \%$. Using the path token to identify content type yields accurate predictions, but, we receive only a small subset of the available URIs.

\begin{tabular}{lrrrr}
\multicolumn{1}{c}{$c_{i}$} & \multicolumn{1}{c}{$H\left(c_{i}\right)$} & \multicolumn{1}{c}{$T P_{c_{i}}$} & $P R_{c_{i}}$ & $R C_{c_{i}}$ \\
\hline text & $11,897,311$ & $10,903,239$ & $91.64 \%$ & $69.55 \%$ \\
appl. & 156,766 & 131.136 & $83.65 \%$ & $9.60 \%$ \\
image & 102,051 & 99,159 & $97.17 \%$ & $59.56 \%$ \\
audio & 19,206 & 9,145 & $47.62 \%$ & $52.25 \%$ \\
video & 5,194 & 1,866 & $35.93 \%$ & $67.88 \%$ \\
message & 130 & 41 & $31.54 \%$ & $3.93 \%$ \\
model & 15 & 14 & $93.33 \%$ & $27.45 \%$ \\
multi. & 82 & 0 & $0.00 \%$ & -
\end{tabular}

\section{Table 6. Results for H3: Content-type header field}

\subsubsection{H3: Content-type header field}

To check if web servers send correct Content-type header fields we compare the Content-type header field $H\left(c_{i}\right)$ with the real content type $F\left(c_{i}\right)$. For computing precision and recall we use: $T P_{c_{i}}=F\left(c_{i}\right) \cap H\left(c_{i}\right)$, $F N_{c_{i}}=F\left(c_{i}\right) \backslash T P_{c_{i}}$ and $F P_{c_{i}}=H\left(c_{i}\right) \backslash T P_{c_{i}}$. Table 6 shows the results of the analysis. The results are similar to H1. Precision values for the content types text, application, image, model and audio are reasonably high, which means that the header information, if available, gives a reasonably good estimate for the real content type. Please observe that not all HTTP responses included a Content-type header, which can be seen from the recall. For the content types audio, video and multipart there are more header fields than the detected real media types, which might be due to the web servers sending incorrect Content-type header fields.

\subsubsection{H4: Document Tree Position}

To prove H4, we extracted over $630 \mathrm{~m}$ URIs from the corpus. Given our restricted resources, we chose not to download the files and use file to determine the real media type. Instead, we used the outcome of H1 and the mapping file to estimate the content type of the linked URIs, which yielded media type estimates for $377 \mathrm{~m}$ URIs, more than half of the extracted links. To check if the position of a link is an indicator for the media type we mapped the links to their content type, which is $L_{T}\left(c_{i}\right)$. For computing precision and recall we use: $T P_{c_{i}}(T)=E\left(c_{i}\right) \cap L_{T}\left(c_{i}\right)$, $F N_{c_{i}}=F\left(c_{i}\right) \backslash T P_{c_{i}}(T)$ and $F P_{c_{i}}=L_{T}(C) \backslash T P_{c_{i}}(T)$.

We observed a correlation between the position of the link in the HTML document and the content type. The relevant HTML element for the content type application are the $<$ LINK>, $\angle O B J E C T>$ and $<$ PARAM $>$ elements (precision value over 45\%). Files of content type image can be extracted from <IMG $>$ and $<$ INPUT $>$ elements with a precision of more than $99.5 \%$. Documents of content 
types audio and video are linked using the $\angle O B J E C T>$ element with a precision of $18 \%$ and the $<$ PARAM $>$ element with a precision of $5 \%$. The low precision values for video has two possible causes: either the use of $\mathrm{H} 1$ skews the results of the file type detection, or JavaScript code obfuscation methods are used at the content provider side to deter crawlers from harvesting video links. Please observe that the highest recall for content type image is at the <IMG> element, for content type application is at the $<\mathrm{LINK}>$ element, and the rest of the content types show the highest recall in the $<A>$ element.

\section{Discussion and Recommendation}

In this section we discuss the result for our hypotheses and give recommendations for a web crawler attempting to fetch only documents of a certain media type.

The test of hypothesis $\mathbf{H 1}$ shows that the file extension of a URI is only correctly used in less than $85 \%$ of the cases, with the highest precision occurring for the content type image $(84.52 \%)$. This implies that more than $15 \%$ of the URIs have a wrong file extention, and especially for the content type video over $79 \%$ of the file extensions are incorrect. For the test of hypothesis H3 we can observe similar effects. The response header fields Content-type only partially match the real determined content types. Again, the content type image has the highest precision with $97.17 \%$. Compared to H1, the response header Content-type fields match more often with the real content type. Furthermore, we can see the path tokens can be used as accurate identifiers for the content types behind a URI, as the precision measure shows in hypothesis test $\mathbf{H 2}$. Moreover, the recall for the content type application is in general less than $10 \%$ for all tests, except for links in the HTML elements html/head/link (45\%), html/body//object (49\%) and html/body/ / param (71\%).

Another observable fact is that for hypothesis tests H1, H3 and H4 the content types audio, video and message showed the lowest precisions. We gave an explanation for each of the hypothesis tests, but another reasonable explanation could be that the Unix tool file might not include the latest video and audio formats. This issue surfaced when we manually verified the content types. For some randomly selected video and audio files from our corpus, the media type returned by the Unix tool file were either empty results or the media type application/octet-stream. Future work is to check the up-to-dateness of the Unix tool file and the dependant libraries.

\begin{tabular}{lcc}
$c_{i}$ & Precision $(>\mathbf{7 5 \%})$ & Recall $(>\mathbf{7 5 \%})$ \\
\hline text & $\mathrm{H} 1, \mathrm{H} 2, \mathrm{H} 3, \mathrm{H} 4$ & $\mathrm{H} 4$ \\
application & $\mathrm{H} 1, \mathrm{H} 2, \mathrm{H} 3$ & - \\
image & $\mathrm{H} 1, \mathrm{H} 2, \mathrm{H} 3, \mathrm{H} 4$ & $\mathrm{H} 1, \mathrm{H} 4$ \\
audio & $\mathrm{H} 2$ & $\mathrm{H} 1, \mathrm{H} 4$ \\
video & $\mathrm{H} 2$ & $\mathrm{H} 4$ \\
message & $\mathrm{H} 2$ & - \\
model & $\mathrm{H} 1, \mathrm{H} 3$ & $\mathrm{H} 1, \mathrm{H} 2, \mathrm{H} 4$ \\
multipart & $\mathrm{H} 2$ & $\mathrm{H} 2, \mathrm{H} 4$
\end{tabular}

\section{Table 7. Summary of results for the four hy- potheses}

\subsection{Recommendation}

Our recommendations are predicated by the goals of the crawler and dependant on the targeted content type and the available information. The goal of the crawling process is to download only files of a certain content type, or in other words achieving high precision during the crawl. Our recommendations are the following, and based on the assumption that the required precision is higher than $75 \%$ :

For the content types text, application, image and model we recommend to use, if available, the file extension and the Content - type header field as identifiers. Furthermore, the position of the link HTML element is a very good identifier for the content types text and image. The path tokens can be used as an identifier for all of the eight content types.

Table 7 shows a summary based on the hypotheses of our recommendations.

\section{Related Work}

To the best of our knowledge, this is the first study about discovering hints identifying media types of files behind URIs.

Most of the papers concerned with web content analysis contain statistics about the distribution of content size, top$\mathrm{k}$ media types, top level domains, and HTTP status codes. The data set presented in [14] consists of $815 \mathrm{~m}$ documents while [10] analysed a corpus of $75 \mathrm{~m}$ URIs. [7] characterises the community web of the people in Portugal based on $3.2 \mathrm{~m}$ pages in 2005. [13] analyses the accessibility of information indexed by search engines and presents estimates such as number of online servers on the Web and the number of files and links per domain. In contrast to presenting general statistics about the data sets used, our work examines identifiers for media types and the correctness of the recommended indicators for media types, such as file exten- 
sion or header Content - type field. Surveys focusing on content metrics exist for media types application/xml or application/rdf+xml; e.g. [6] analysed the RDF Web in 2002, [16] published a survey of the OWL ontology landscape in 2006, [5] analysed RDF and OWL documents based on $1.7 \mathrm{~m}$ sources in 2006, and [1] studied the usage of web services in 2006. Again, these studies do not present information about identifiers that can be used to determine the content type of a given URI.

\section{Conclusion}

We analysed a representative subset of the Web consisting of $22 \mathrm{~m}$ files and $630 \mathrm{~m}$ URIs. Comparing our statistics to previously published analysis (from 2002 [14], from 2005 [7]), we conclude that the Web has not significantly changed over the last six years in terms of response code, top level domain, and media type distribution.

We addressed the problem of discovering URIs of particular content types by postulating and testing four hypotheses. The result of the evaluation establishes that for the content types text, image, and application, the recommendations from RFCs (file extension and header content type) and W3C (link position in HTML) are almost being followed, while results for audio and video are much less consistent. In addition, single path tokens of a URI give a cue to the content type of the file behind the URI. Furthermore, we gave recommendations to assist a crawler traversing the Web for certain media types. Our findings and recommendations can support web crawler developers in implementing heuristics for efficient discovery of structured content on the Web and can help media type targeted search engines to solve the problem of gathering files of a certain media type from the Web.

\section{Acknowledgements}

This work has been supported by Science Foundation Ireland under project Lion (SFI/02/CE1/I131).

\section{References}

[1] D. Bachlechner, K. Siorpaes, H. Lausen, and D. Fensel. Web service discovery - a reality check. In Proceedings of the 1st Workshop: SemWiki2006 - From Wiki to Semantics, colocated ESWC, Budva, Montenegro, June 2006.

[2] S. Chakrabarti. Mining the Web: Discovering Knowledge from Hypertext Data. Morgan-Kauffman, 2003.

[3] S. Chakrabarti, M. van den Berg, and B. Dom. Focused crawling: A new approach to topic-specific web resource discovery. Computer Networks, 31(11-16):16231640, 1999.
[4] M. Diligenti, F. Coetzee, S. Lawrence, C. L. Giles, and M. Gori. Focused crawling using context graphs. In Proceedings of the 26th International Conference on Very Large Databases (VLDB 2000), pages 527-534, 2000.

[5] L. Ding and T. Finin. Characterizing the semantic web on the web. In Proceedings of the 5th International Semantic Web Conference, Athens, Georgia, November 2006.

[6] A. Eberhart. Survey of rdf data on the web. Technical report, International University in Germany, 2002.

[7] D. Gomes and M. J. Silva. Characterizing a national community web. ACM Transactions on Internet Technology, 5(3):508-531, 2005.

[8] A. Gulli and A. Signorini. The indexable web is more than 11.5 billion pages. In $W W W$ '05: Posters of the 14th international conference on World Wide Web, pages 902-903, New York, NY, USA, 2005. ACM.

[9] A. Harth, J. Umbrich, and S. Decker. Multicrawler: A pipelined architecture for crawling and indexing semantic web data. In International Semantic Web Conference, pages 258-271, 2006.

[10] A. Heydon and M. Najork. Mercator: A scalable, extensible web crawler. World Wide Web, 2(4):219-229, 1978.

[11] M. Jamali, H. Sayyadi, B. B. Hariri, and H. Abolhassani. A method for focused crawling using combination of link structure and content similarity. In Web Intelligence, pages 753-756. IEEE Computer Society, 2006.

[12] H. Lausen and T. Haselwanter. Finding web services. In 1st European Semantic Technology Conference, volume 2007. ESTC, June 2007.

[13] S. Lawrence and C. L. Giles. Accessibility of information on the web. Intelligence, 11(1):32-39, 2000.

[14] M. Najork and A. Heydon. High-performance web crawling. In Handbook of massive data sets, pages 25-45, Norwell, MA, USA, 2002. Kluwer Academic Publishers.

[15] G. Pant, P. Srinivasan, and F. Menczer. Crawling the web, 2004.

[16] T. D. Wang, B. Parsia, and J. Hendler. A survey of the web ontology landscape. In Proceedings of the 5th International Semantic Web Conference, Athens, Georgia, November 2006. 